\title{
A time of renewal
}

\author{
Domingo M. BRAILE*
}

$\mathrm{E}$ aster, with all its symbolism for the Christian world, brings an opportunity to reflect on our attitudes and is a great opportunity to renew behaviors and ideas. Taking the opportunity of the proximity of this date, on behalf of the Editorial Board of the Brazilian Journal of Cardiovascular Surgery (BJCVS), I present some new features, implemented and under implementation since the beginning of the year, always with the aim of the constantly improve and to keep us pari passu with the scientific publications of greatest impact.

Our website (www.rbccv.org.br) online since 2005, had presented some problems for navigation, due to the significant increase in the number of hits - a result from the indexing in ISI and Medline. In 2009, only in February, an atypical month, there were 44,738 hits, an average nearly 1,600 hits daily. If we add to this number the visits to the Scielo website (www.scielo.br/RBCCV), it will be almost 82 thousand hits!

To solve this problem and avoid annoyance to users, the BJCVS website, hosted at Hospedagem Profissional plan, by Locaweb, was relocated, under the supervision of GN1, which manages the site, to the Cloud Computing plan, with benefits such as those mentioned below:

1 - Supporting more simultaneous hits: differently from the professional hosting on which several sites shared resources from the server, at Cloud Computing multiple servers share computing resources to one site.

2 - Greater control over the growth of server usage: in the previous plan, growth in the amount of hits and server usage was detected only when the system fell. At Cloud, there is a monitor that displays the amount of computational resources used by the site. Thus, one can predict when the system will begin to become unstable and to expand the computational resources to keep the site functional.

3 - Better performance: in addition to stability in the hosting service, performance was improved, so the loading time of pages was reduced considerably.

The system may have some instability in the first months of operation and, since now, I ask my colleagues who use the site to communicate us about any problems in order to solve them in the shortest possible time.

Another new is that, in order to increase the number of collaborator from BJCVS, I've asked the members of Editorial Board and Editors to indicate colleagues to join the list of reviewers. It was not surprise to me the exceptional return, a further evidence of the commitment of the Editorial Board with our jounal. There were over 40 nominees, and the majority has already accepted the invitation and will perform evaluations soon. These new reviewers who will be added to those who were already pushing our journal are very important to give an impetus to the publication, because many of the new reviewers are young surgeons, but with solid academic and practical experience.

Even within this spirit of change, I ask all those who wish to submit manuscripts to insert directly on our site and do not forward them by e-mail, as it demands work to be inserted into the system, causing delays in the procedure - which we hope occurs as fast as possible. The Executive Editor, Ricardo Brandau, and the team of GN1 are available to clarify any doubts. I count on the usual collaboration of all. Although we are working hard, we have some obstacles to overcome. Since last year, I reported the dissatisfaction of Editors of Scientific Journals with criteria from CAPES for the reclassification of publications by QUALIS. These criteria are questionable and, even after the explanations given by representatives of the Institution at meetings held last year, led by AMB - Brazilian Medical Association, it is not clear, for example, why the Brazilian Archives of Cardiology, indexed in these databases of BJCVS, was classified as B3 while BJCVS was "reclassified” as B4.

On page III of this edition, we published an editorial from AMB, signed by 60 editors and published simultaneously in 60 Brazilian journals, which shows a summary of conversations held with CAPES and asking to change the criteria for classification of journals by the bodies responsible. I ask the reader the carefully reading of such Editorial, because the future of our journals depends greatly on the political will of CAPES. In my opinion, the dilemma is simple: We can simply internationalizing Brazilian articles, publishing them in foreign journals of the first world, or we can internationalize the good Brazilian journals, showing the world that we are a scientifically developed country. I am absolutely sure this is very possible!

I know that the subject is complex and will not be solved overnight, but it is of utmost importance that Editors continue discussions and managements with CAPES, so that we can reach an agreement that may satisfy both parties.

Whether or not I agree with the criteria of CAPES, we 
must strive to increase our impact factor. This is possible only if the articles published in BJCVS are cited more and for that it is needed to be of scientific value even better. Thus, reviewers should be even more rigorous in their evaluations.

In this edition, we have more six articles available for testing by the Continuing Medical Education (CME) system: "Concepts of basic physics that every cardiovascular surgeon should know. Part 1 - Mechanics of fluids”, page 1; “Assessment of the EuroSCORE as a predictor for mortality in valve cardiac surgery at the Heart Institute of Pernambuco", page 11; "Hospital mortality in surgery for reconstruction of the right ventricular outflow tract with pulmonary homograft”, page 25; “Use of nifedipine and acute kidney injury incidence in postoperative of myocardial revascularization surgery with cardiopulmonary bypass”, page 32; “Comparison study of two different patient-controlled anesthesia regiments after cardiac surgery”, page 38; and “The effects of modified ultrafiltration on pulmonary function and transfusion requirements in patients undergone coronary artery bypass surgery”, page 59.

Also, I would like to highlight the Editorial by the Associate Editor Ulisses Croti (page VIII), relating the integration of the Cardiac Surgery Service of the São José do Rio Preto Medical School with the Children's HeartLink, an NGO dedicated to supporting the development, training and mobilizing people and institutions to help children with heart defects worldwide. This NGO has already operated in many countries, especially in the East, and finally arrived in Brazil in September 2009, and chosen the Institution in São José do Rio Preto due to the characteristics presented. The first joint activity lasted a week and reached full success.

In January, we received the editorial supporting of CNPq, of R $\$ 35.000,00$ thousand Reais, which will be used to pay the costs of formatting and diagramation, and also services to improve the website. Although far from ideal, the amount is always important to maintain and raise our standard of quality.

In the next edition I will approach the $37^{\text {th }}$ Congress of BSCVS, held in Belém, PA, that has, as usual, a Supplement from BJCVS with all the summaries of presentations, which now belong to the annals of Congresses published in an indexed journal.

My warmest regards!

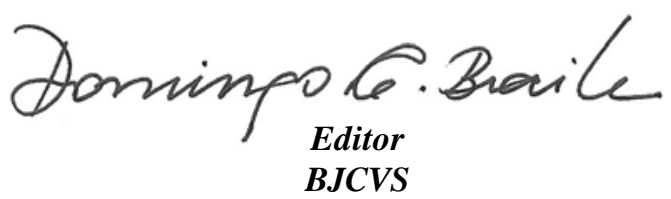

